\title{
Protuberant Electrode Structures for New Retinal Implants ${ }^{\dagger}$
}

\author{
Pedro González Losada 1,*, Lionel Rousseau ${ }^{1}$, Marjorie Grzeskowiak ${ }^{1}$, Julie Degardin ${ }^{2}$ \\ Sarah Berhanu ${ }^{2}$, Elisabeth Dubus ${ }^{2}$, Serge Picaud ${ }^{2}$ and Gaëlle Lissorgues ${ }^{1}$ \\ 1 University Paris Est, ESYCOM-ESIEE-MLV, France; lionel.rousseau@esiee.fr (L.R.); \\ marjorie.grzeskowiak@univ-mlv.fr (M.G.); gaelle.lissorgues@esiee.fr (G.L.) \\ 2 Institute of Vision, Paris, France; julie.degardin@inserm.fr (J.D.); sarah.berhanu@inserm.fr (S.B.); \\ elisabeth.dubus@inserm.fr (E.D.); serge.picaud@inserm.fr (S.P.) \\ * Correspondence: pedro.gonzalezlosada@esiee.fr; Tel.: +33-641-281-517 \\ † Presented at the Eurosensors 2017 Conference, Paris, France, 3-6 September 2017.
}

Published: 9 August 2017

\begin{abstract}
Focal stimulation of neural tissue is a challenge in retinal prosthetic devices in which the aim is to improve the spatial resolution of the stimulation and therefore increase the electrode density of the prosthetic devices. Our work intends to create a new implant able to enhance the focalisation of the stimulation signal through protuberant electrodes. These electrodes are micro fabricated on a soft polyimide substrate using classical metal electrodeposition techniques. Before proceeding with fabrication a FEM model of the electrode's current density was done to select the best-performing structures and geometries in terms of local stimulation. Based on these models, several prototypes were fabricated and implanted in vivo into a rat's eye to verify the adaptation to the retina tissue.
\end{abstract}

Keywords: microfabrication; retinal prostheses; implantable device; 3D electrode; retinal stimulation

\section{Introduction}

Work already carried out in retinal implants shows that several geometric modifications can be made. On the one hand, the use of a ground plane around the electrodes instead of a far return electrode keeps the stimulation signal more localised on the electrode area [1]. On the other hand, the insertion of the electrode in a cavity of few micrometres surrounded by a ground plane creates 3D shapes which represents an improvement [2]. An important aspect is the interaction between the electrode and the neural tissue: a closer interaction will entail a better focalisation. To obtain such effect, 3D mushroom or pillar electrodes have been used in Micro Electrode Arrays to improve the interaction between cultivated cells and the electronic device [3].

Our work tries to integrate both elements in a soft implant to study two main aspects: the performance of the electrical stimulation and the inner integration of the implant with the tissue in vivo. We carried out our research in three phases. First we developed a FEM model of the electrode with different geometries to evaluate its current delivering performance. Then, taking into account these results and using the planar implant fabrication technology previously developed in our laboratory, we added new fabrication steps to build the protuberant electrodes. Finally, in order to verify the adaptation of the retinal tissue to these protuberant shapes, the devices were implanted in sub-retinal position into $\mathrm{P} 23 \mathrm{H}$ blind rats with a very thin retina, similar to the one that could be found in the targeted pathologies. 


\section{Materials and Methods}

\subsection{FEM Simulation}

A finite element model (FEM) has been developed using COMSOL. The geometry consists of a single protuberant metallic electrode embedded in an insulating substrate and surrounded by a liquid environment with a conductivity which represents the physiological environment. The interface between the insulating substrate and the liquid environment is modelled using insulating boundaries described by Equation (1). For the electrode-electrolyte interface, robin boundary condition described by Equation (2) is used as previously defined by Joucla et al. [1]. The conductivity values for the different materials are considered homogeneous and isotropic, as summarised in Table 1.

Two types of structures are modelled for comparison: a flat electrode in a cavity surrounded by a ground plane and a single protuberant electrode in a cavity surrounded by a ground plane, with the same cavity depth.

$$
\begin{gathered}
\sigma \nabla \mathrm{V} \boldsymbol{n}=0, \\
\sigma \nabla \mathrm{V} \boldsymbol{n}=\mathrm{g}\left(V_{\text {metal }}-V\right),
\end{gathered}
$$

Table 1. Parameters used for the simulation.

\begin{tabular}{ccc}
\hline Material & Conductivity (S/m) & Relative Permittivity \\
\hline Physiological liquid & $1.47 \times 10^{-2}$ & 81 \\
Insulating material & $1.31 \times 10^{-18}$ & 3.4 \\
Conductive material & $45.6 \times 10^{6}$ & - \\
\hline
\end{tabular}

\subsection{Microfabrication}

As mentioned in the introduction, the fabrication technology is based on the planar implant technology to which new steps haven added in order to put in the 3D shaped wells the protuberant electrodes. The size of the hexagonal cavities is $100 \mu \mathrm{m}$ and the diameter of the protuberances is $12 \mathrm{um}$. We designed different cavities in the same implant: some of them empty, and the others with $1,2,3$ or 4 protuberant structures. The fabrication process for the soft implants on a silicon wafer is the following: After the deposition of a sacrificial layer composed of titanium $(100 \mathrm{~nm})$ and aluminium (500 nm), $10 \mu \mathrm{m}$ of polyimide (PI 2611) are spin coated. Layers of titanium (100 nm) and gold $(500 \mathrm{~nm})$ are deposited by sputtering and patterned by photolithography to define electrodes, tracks and pads. Finally, SU8 2002 (MicroChem) is spin coated to form a $2 \mu \mathrm{m}$ passivation layer. Using photolithography, openings are created on the pads and electrodes. Then, $500 \mathrm{~nm}$ of aluminium is sputtered to define the shape of the implant by lithography. To etch the polymer, we use reactive ion plasma composed of a mix of Ar and $0_{2}$ at 120 Watt. To peel off the implants, the aluminium layer is dissolved by electro erosion

In our case, we do not peel off the implants and continue the fabrication to produce 3D structures. On the whole wafer, we deposit a thin layer of titanium $(50 \mathrm{~nm})$ and $150 \mathrm{~nm}$ of copper corresponding to the seed layer. A photoresist is deposited to form the mould for protuberant electrodes. The wafer is placed in a copper solution and a current of $100 \mathrm{~mA}$ is applied to create copper protuberances. The photoresist is cleaned in solvent and a thick photoresist $(15 \mu \mathrm{m})$ is used to perform the counter ground plane. Once the photolithography step is completed, the wafer is placed for a second time in the copper electrodeposition solution in the same growth conditions. Wafers are cleaned and the initial seed layer is etched. A photoresist protection is applied before peeling off the implants. After cleaning, a thin layer of $2 \mu \mathrm{m}$ of parylene $C$ is used to encapsulate the whole copper structure (as Copper is not biocompatible). For the next generation of implants, the 3D structures will be built through gold electrodeposition (for biocompatibility). 


\subsection{Implantation}

The implantation of the devices is done in $\mathrm{P} 23 \mathrm{H} / \mathrm{HO}$ rats at the age of 9 months and only in one eye. The placement of the implant on the sub-retinal position is checked periodically during the implantation time by means of eye fundus observation (Micron III) and OCT images. After 19 weeks the rats are sacrificed, the retina is explanted and cell labelling is performed.

\section{Results}

\subsection{FEM Simulation}

The cross sectional view of the current density is shown in Figure 1 for the planar geometry (a) and protuberant one (b). In the planar geometry a border effect is identified both in the border of the electrode and the ground plane: most of the current penetrates into the liquid in this part of the electrode. In the protuberant geometry, we appreciate a redistribution of the current density as the border effect still exists but part of the current flows to the media through the protuberance.

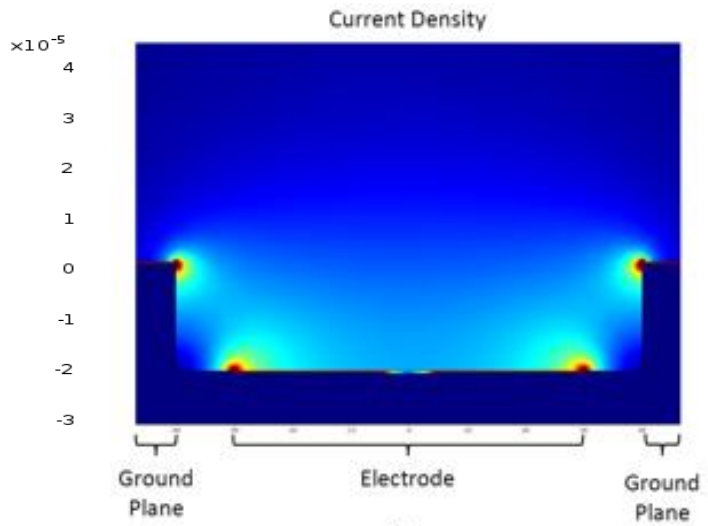

(a)

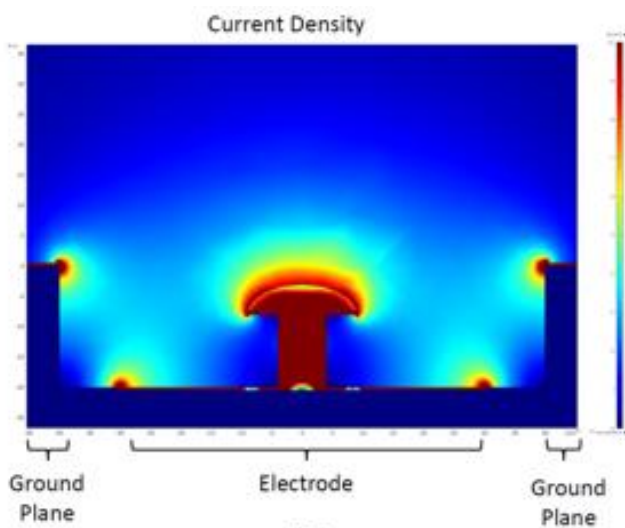

(b)

Figure 1. Comparison of the current density delivered by a flat electrode and a protuberant electrode in a two level configuration: ground plane and flat electrode plane are not at the same level-same colour scale.

\subsection{Microfabrication}

In order to characterise the electrodeposition time for the protuberant electrodes and the ground plane, several tests were done on wafers where only the electrodes or the ground plane were patterned. The time and the thickness of the metallic structures were measured using a Wykko profilometer to obtain the relation between the electrodeposition time and the electrodeposed metal thickness. These values were used for the electrodeposition of the structures on the soft implants and the results are summarised in Table 2.

Table 2. Relation between the electrodeposition time and the thickness achieved.

\begin{tabular}{ccccc}
\hline & \multicolumn{2}{c}{ Electrode } & \multicolumn{2}{c}{ Ground Plane } \\
\hline Wafer & Time [min] & Thickness [um] & Time [min] & Thickness [um] \\
\hline W1 & 17 & 3.25 & 57 & 23.00 \\
W2 & 17 & 3.20 & 50 & 22.00 \\
\hline
\end{tabular}

The surface of the electrodes was examined using a scanning electron microscope (ZEISS NEON40) to see the general aspect of the implant (Figure 2a) and the aspect of the individual electrodes (Figure 2b). 


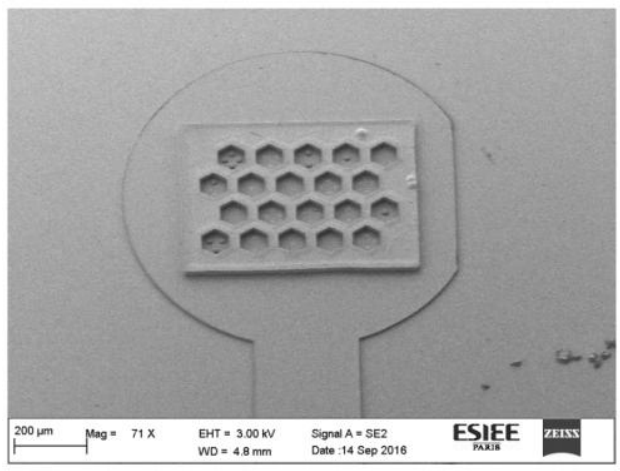

(a)

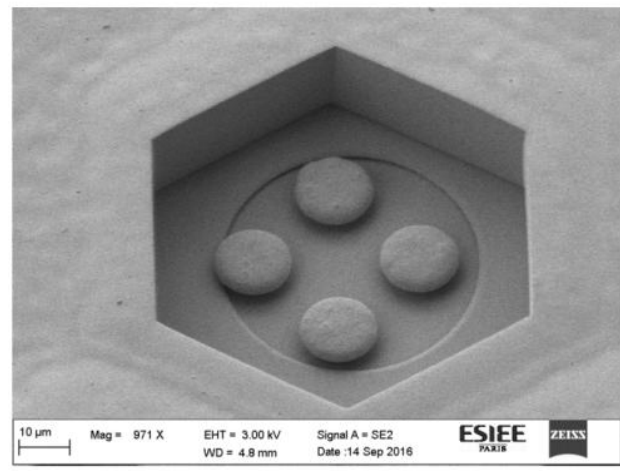

(b)

Figure 2. SEM image of the implant (a) General view of the implant head on soft substrate; (b) Detail of the configuration consisting of 4 protuberant electrodes embedded in the well formed by the ground plane.

\subsection{Implantation}

The digital endoscope (Figure 3a) and OCT images taken during the whole implantation period can show that the implant is properly placed in the sub-retinal position. A confocal analysis of the explanted tissue allows us to check the adaptation of the retina to the new implant geometry. The images obtained (Figure $3 b$ ) do not show a glial reaction of the tissue around the implant and the cells can descent into the cavities whether there are protuberances or not.

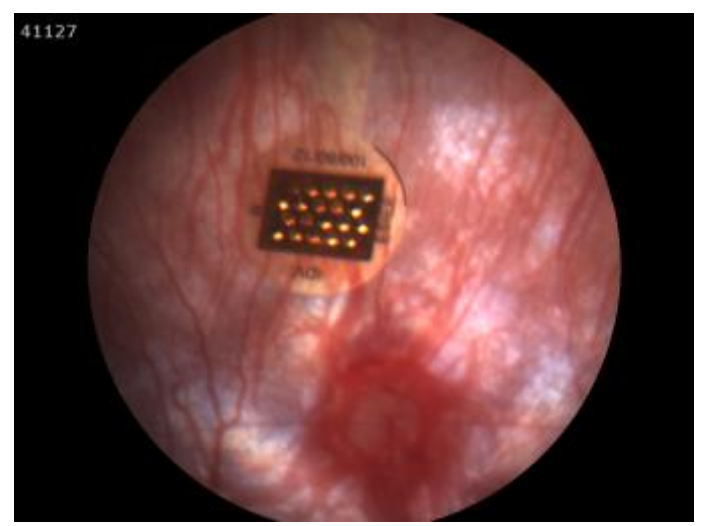

(a)

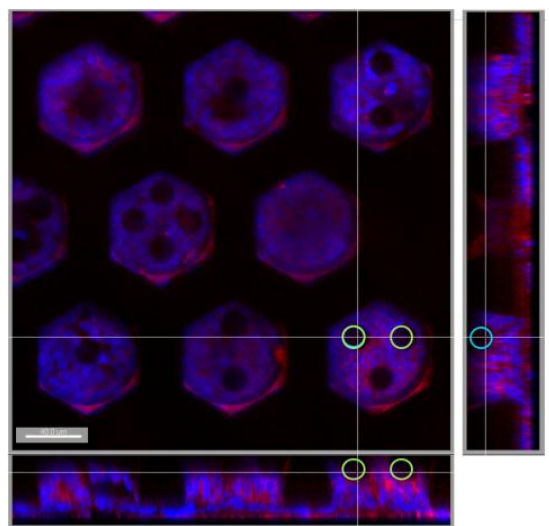

(b)

Figure 3. (a) Verification of the correct placing of the implant in sub-retinal position two weeks after implantation: Eye fundus of $\mathrm{P} 23 \mathrm{H}$ rats with the implant obtained with a Micron III; (b) Confocal image of the retinal tissue and implant, where blue cells represent the bipolar cells after 19 weeks.

\section{Conclusions}

Focal stimulation is a challenge for neural prostheses and especially for retinal ones. Improvement of the stimulation process can be achieved by modifying several parameters as the type of stimulation signal, the materials used for the prostheses fabrication or their geometry. We explore here this last option to try to improve the interface between the electrode and the tissue. For this purpose we first developed a FEM model of the electrode that we could micro fabricate and which gave us an idea of the best structures to explore. After micro fabrication, we implanted them in a PH23 rat's eye to examine the adaptation of the retinal tissue to this new 3D geometry. Images obtained using a confocal microscope and specific labelling showed that the retina is able to adapt to the shape of this new implant and that bipolar cells are present in the cavities.

Acknowledgments: Work performed within the ANR grant project NEUROMEDDLE.

Conflicts of Interest: The authors declare no conflict of interest. 


\section{References}

1. Joucla, S.; Yvert, B. Improved focalization of electrical microstimulation using microelectrode arrays: A modeling study. PLOS ONE 2009, 4, e4828.

2. Djilas, M.; Olès, C.; Lorach, H.; Bendali, A.; Dégardin, J.; Dubus, E.; Lissorgues-Bazin, G.; Rousseau, L.; Benosman, R.; Ieng, S.; et al. Three-dimensional electrode arrays for retinal prostheses: Modeling, geometry optimization and experimental validation. J. Neural Eng. 2011, 8, 046020.

3. Huys, R.; Braeken, D.; Van Meerbergen, B.; Winters, K.; Eberle, W.; Loo, J.; Tsvetanova, D.; Chen, C.; Severi, S.; Yitzchaik, S.; et al. Novel concepts for improved communication between nerve cells and silicon electronic devices. Solid State Electron. 2008, 52, 533-539.

(C) 2017 by the authors. Licensee MDPI, Basel, Switzerland. This article is an open access article distributed under the terms and conditions of the Creative Commons Attribution (CC BY) license (http://creativecommons.org/licenses/by/4.0/). 